\title{
On the existence and uniqueness of solutions for fuzzy fractional differential equations
}

\author{
A. Rivaz ${ }^{1}$, O.S. Fard ${ }^{2, *}$ and T.A. Bidgoli ${ }^{3}$ \\ ${ }^{1}$ Department of Applied Mathematics, Faculty of Mathematics and Computer, Shahid Bahonar University of Ker- \\ man, Kerman, Iran \\ ${ }^{2}$ School of Mathematics and Computer Science, Damghan University, Damghan, Iran \\ 3 Department of Applied Mathematics, Faculty of Mathematics and Computer, Shahid Bahonar University of Ker- \\ man, Kerman, Iran \\ *Corresponding Author \\ E-mail: arivaz@mail.uk.ac.ir ${ }^{1}$, osfard@du.ac.ir ${ }^{2}$, omidsfard@gmail.com ${ }^{2}$, tayebeh.aab@gmail.com ${ }^{3}$
}

\begin{abstract}
In this paper, we introduce and define a new metric on the space of fuzzy continuous functions in the fractional calculus. Regarding this metric and using the well-known Banach fixed point theorem, we provide some conditions that guarantee the existence and uniqueness of solution to a nonlinear fuzzy fractional differential equation in the proposed metric. Finally, two examples are given to illustrate the results.
\end{abstract}

2010 Mathematics Subject Classification. 34A08. 34A12

Keywords. Fuzzy Liouville-Caputo's derivative, Metric space, Fuzzy fractional differential equation, Mittag-Leffler functions.

\section{Introduction}

Theory of differential equations is an important part of pure and applied mathematics which plays a prominent role in many disciplines including engineering, physics, economics and biology. One of the significant branches of theory of differential equations is fractional differential equations that in recent years, has received considerable attention not only in mathematical research but also in other applied sciences. In fact, Fractional differential equations are the development of differential equations to real order. These type of differential equations can be applied to many real-world field such as polymer physics, viscoelastic materials, viscous damping and seismic analysis see $[3,9,11,12,13,14,18]$.

On the other hand, when one intends to analyze a real world phenomenon, it is also necessary to deal with uncertain factors. In this situation, the theory of fuzzy sets may be one of the best non-statistical or non-probabilistic approach, which leads us to investigate theory fuzzy fractional differential equations.

Recently, the topic of existence and uniqueness for the solutions to linear and nonlinear fuzzy fractional differential equations has been further investigated and discussed by many researchers in various aspects. For example, in [2] the existence and uniqueness of solutions of Riemann- Liouville fuzzy fractional differential equations has been proved by Arshad and in [16] the existence and uniqueness of solutions as well the approximate solutions to fuzzy fractional differential equations under the Liouville-Caputo H-differentiability has been studied by Salahshour and et al.. Furthermore, the existence and uniqueness of solutions for fuzzy fractional differential equations under the Liouville-Caputo generalized Hukuhara differentiability has been investigated by Allahviranloo et 
al. in [1].

In this paper, we intend to propose a new metric on the space of fuzzy continuous functions by using Mittag-Leffler functions and we study and investigate the existence and uniqueness of solutions to nonlinear fuzzy fractional differential equations under fractional generalized H-differentiability in the sense of the Liouville-Caputo differentiability.

This paper organized as follows: A brief review on the well-known fuzzy concepts as well as fractional calculus are given in Section 2. Afterward in Section 3, a new metric for the space of fuzzy continuous functions is introduced and the main result of the paper i.e., the existence and uniqueness of solutions for nonlinear fuzzy fractional differential equations will be proved. Finally, two examples are given to illustrate the application of the result.

\section{A brief review on fuzzy concepts and fractional calculus}

\subsection{Fuzzy Theory}

Definition 2.1. [5] Let $X$ be a nonempty set. A fuzzy set $u$ in $X$ is characterized by its membership function $u: X \rightarrow[0,1]$. Then, for each $x \in X$ we interpret $u(x)$ as the degree of membership of the element $x$ in the fuzzy set $u: u(x)=0$ corresponded to non membership; $0<u(x)<1$ to partial membership; and $u(x)=1$ to full membership.

Definition 2.2. [5] We denote by $\mathbb{R}_{\mathcal{F}}$ the class of fuzzy subsets of the real axis $u: \mathbb{R} \rightarrow[0,1]$ satisfying the following properties:

(i) $u$ is normal, i.e., there exists $x_{0} \in \mathbb{R}$ with $u\left(x_{0}\right)=1$;

(ii) $u$ is a convex fuzzy set, i.e., $u(\lambda x+(1-\lambda) y) \geq \min \{u(x), u(y)\}$ for all $\lambda \in[0,1]$ and $x, y \in \mathbb{R}$;

(iii) $u$ is upper semicontinuous on $\mathbb{R}$, i.e. for each $a \in[0,1],\{x: u(x)<a\}$ is open in $\mathbb{R}$;

(iv) $\operatorname{cl}\{x \in \mathbb{R}: u(x)>0\}$ is compact, where $c l$ denotes the closure of a set.

We call $\mathbb{R}_{\mathcal{F}}$ the space of fuzzy numbers.

Obviously $\mathbb{R} \subset \mathbb{R}_{\mathcal{F}}$. Here $\mathbb{R} \subset \mathbb{R}_{\mathcal{F}}$ is understood as $\mathbb{R}=\left\{\chi_{\{x\}} ; x\right.$ is usual real number $\}$. For $0<r \leq 1$, denote $[u]^{r}=\{x \in \mathbb{R} ; u(x) \geq r\}$ and $[u]^{0}=\operatorname{cl}\{x \in \mathbb{R} ; u(x)>0\}$.

By the definition of fuzzy numbers, it follows that for any $r \in[0,1],[u]^{r}$ is a bounded closed interval. The notation $[u]^{r}=\left[\underline{u}^{r}, \bar{u}^{r}\right]$ denotes explicitly the $r$-level set of $u$. We refer to $\underline{u}$ and $\bar{u}$ as the lower and upper branches on $u$, respectively.

For $u, v \in \mathbb{R}_{\mathcal{F}}$ and $\lambda \in \mathbb{R}$, the sum of two fuzzy numbers and the multiplication between a real and a fuzzy number are defined respectively by

$$
[u+v]^{r}=[u]^{r}+[v]^{r}=\left\{x+y: x \in[u]^{r}, y \in[v]^{r}\right\}
$$

and

$$
[\beta u]^{r}=\beta[u]^{r}=\left\{\beta x: x \in[u]^{r}\right\}
$$

for all $r \in[0,1]$, where $[u]^{r}+[v]^{r}$ is the usual addition of two intervals of $\mathbb{R}$ and $\beta[u]^{r}$ is the usual product of a number and a subset of $\mathbb{R}$. 
The Hausdorff distance between fuzzy numbers is given by $d_{\infty}: \mathbb{R}_{\mathcal{F}} \times \mathbb{R}_{\mathcal{F}} \rightarrow \mathbb{R}^{+} \cup\{0\}$ as $d_{\infty}(u, v)=\sup _{0<r<1} \max \left\{\left|\underline{u}^{r}-\underline{v}^{r}\right|,\left|\bar{u}^{r}-\bar{v}^{r}\right|\right\}$. The metric space $\left(\mathbb{R}_{\mathcal{F}}, d_{\infty}\right)$ is complete and the following properties for the metric $d_{\infty}$ hold (see [15]):

(i) $d_{\infty}(u+w, v+w)=d_{\infty}(u, v), \quad \forall u, v, w \in \mathbb{R}_{\mathcal{F}} ;$

(ii) $d_{\infty}(u+w, v+z) \leq d_{\infty}(u, v)+d_{\infty}(w, z) \quad \forall u, v, w, z \in \mathbb{R}_{\mathcal{F}}$;

(iii) $d_{\infty}(k u, k v)=|k| d_{\infty}(u, v), \quad \forall k \in \mathbb{R}, u, v \in \mathbb{R}_{\mathcal{F}}$.

Definition 2.3. [17] A mapping $f: T \times \mathbb{R}_{\mathcal{F}} \rightarrow \mathbb{R}_{\mathcal{F}}$ is called continuous at $\left(t_{0}, x_{0}\right) \in T \times \mathbb{R}_{\mathcal{F}}$ provided for any arbitrary $\varepsilon>0$, there exist an $\delta(\varepsilon)>0$ such that

$$
d_{\infty}(f(t, x)), f\left(t_{0}, x_{0}\right)<\varepsilon,
$$

whenever $\left|t-t_{0}\right|<\delta(\varepsilon)$ and $d_{\infty}\left(x, x_{0}\right)<\delta(\varepsilon)$ for all $t \in T, x \in \mathbb{R}_{\mathcal{F}}$.

Definition 2.4. [6] Let $x, y \in \mathbb{R}_{\mathcal{F}}$. If there exists $z \in \mathbb{R}_{\mathcal{F}}$ such that $x=y+z$, then $z$ is called the $H$-difference of $x$ and $y$ and it is denoted by $x \ominus y$.

Definition 2.5. $[7,19]$ Given $u, v \in \mathbb{R}_{\mathcal{F}}$, the $g H$-difference is the fuzzy number $w$, if it exists, such that

$$
u \ominus_{g H} v=w \Leftrightarrow \quad(i) u=v+w \quad \text { or } \quad(i i) v=u+(-1) \cdot w
$$

Remark 2.6. If $u \ominus_{g H} v$ exists, then its $r$-level set is given by

$$
\left[u \ominus_{g H} v\right]^{r}=\left[\min \left\{\underline{u}^{r}-\underline{v}^{r}, \bar{u}^{r}-\bar{v}^{r}\right\}, \max \left\{\underline{u}^{r}-\underline{v}^{r}, \bar{u}^{r}-\bar{v}^{r}\right\}\right] .
$$

Definition 2.7. [1] The generalized Hukuhara derivative of a fuzzy-valued function $f:(a, b) \rightarrow \mathbb{R}_{\mathcal{F}}$ at $x_{0}$ is defined as

$$
f_{g H}^{\prime}\left(x_{0}\right)=\lim _{h \rightarrow 0} \frac{f\left(x_{0}+h\right) \ominus_{g H} f\left(x_{0}\right)}{h}
$$

If $f_{g H}^{\prime}\left(x_{0}\right) \in \mathbb{R}_{\mathcal{F}}$, is said that $f$ is $g H$-differentiable at $x_{0}$.

Remark 2.8. If $f\left(x_{0}+h\right) \ominus_{g H} f\left(x_{0}\right)$ exists as $(i)$ in $(1)$ is said that $f_{g H}^{\prime}\left(x_{0}\right)$ is $[(i)-g H]-$ differentiable and If it exists as $(i i)$ in $(1)$ is said that $f_{g H}^{\prime}\left(x_{0}\right)$ is $[(i i)-g H]-$ differentiable.

\section{$2.2 \quad$ Fractional calculus}

The Rimann-Liouville fractional derivative and integral of order $\alpha>0$ of a function $y$, respectively are defined by (see [8])

$$
D^{\alpha} y(t):=\frac{1}{\Gamma(\lceil\alpha\rceil-\alpha)} \frac{d^{\lceil\alpha\rceil}}{d t^{\lceil\alpha\rceil}}\left(\int_{0}^{t}(t-s)^{\lceil\alpha\rceil-1-\alpha} y(s) d s\right)
$$

and

$$
I^{\alpha} y(t):=\frac{1}{\Gamma(\alpha)} \int_{0}^{t}(t-s)^{\alpha-1} y(s) d s
$$


besides, for $\alpha=0$, we set $D^{0}:=I$, the identity operator.

Also the Liouville-Caputo derivative is defined by

$$
{ }^{C} D^{\alpha} y(t):=D^{\alpha}\left(y-T_{\lceil\alpha\rceil-1}[y]\right)(t),
$$

where $T_{\lceil\alpha\rceil-1}[y]$ is the Maclaurin polynomial of order $\lceil\alpha\rceil-1$ of $y=y(t)$, i.e., $T_{\lceil\alpha\rceil-1}[y](t)=$ $\sum_{k=0}^{m-1} \frac{y^{k}(0)}{k !} t^{k}$

In the fractional calculus, the Mittag-Leffler function plays an important role same as the exponential function in the classical calculus. The Mittag-Leffler function of order $\alpha>0$ is denoted by $E_{\alpha}(z)$ and it is defined as follows

$$
E_{\alpha}(z):=\sum_{k=0}^{\infty} \frac{z^{k}}{\Gamma(\alpha k+1)}, \quad \alpha>0, z \in \mathcal{C}
$$

Note that, for $\alpha=1$ the function $E_{1}(z)$ becomes the classical exponential function $\exp (z)$. Moreover, by composing two functions $E_{\alpha}(z)$ and $t^{\alpha}$ we have

$$
E_{\alpha}\left(\lambda t^{\alpha}\right):=\sum_{k=0}^{\infty} \frac{\left(\lambda t^{\alpha}\right)^{k}}{\Gamma(\alpha k+1)}, \quad t \in[0, a] \subset \mathbb{R}
$$

where $\alpha>0$ and $\lambda>0$ is a constant. In fact, the function $E_{\alpha}\left(\lambda t^{\alpha}\right)$ is the unique solution to the following initial value problem

$$
\begin{aligned}
& { }^{C} D^{\alpha} x(t):=D^{\alpha}\left(x-T_{\lceil\alpha]-1}[x]\right)(t)=\lambda x(t), \\
& x(0)=1, x^{\prime}(0)=0, \ldots, x^{\lceil\alpha\rceil-1}(0)=0,
\end{aligned}
$$

for $t \geq 0$.

Here, it is necessary to review that for $\alpha \in \mathbb{N},\lceil\alpha\rceil=\alpha$,

$$
{ }^{C} D^{\alpha} f=D^{\alpha}\left(f-T_{\alpha-1}[f]\right)=D^{\alpha} f-D^{\alpha}\left(T_{\alpha-1}[f]\right)=D^{\alpha} f
$$

since $T_{\alpha-1}[f]$ is a polynomial of degree $\alpha-1$.

\section{The main result}

We start this section with the following definition of Liouville-Caputo's derivative under generalized Hukuhara derivative presented in [1].

Definition 3.1. Let $f_{g H}^{(m)} \in C[a, b] \cap L[a, b]$. The $g H$ - fractional Liouville-Caputo differentiability of fuzzy valued function $f$ is defined as follows:

$$
{ }_{g H}^{C H} D^{\alpha} f(x)=I^{m-\alpha}\left(f_{g H}^{(\lceil\alpha\rceil)}\right)(x)=\frac{1}{\Gamma(\lceil\alpha\rceil-\alpha)} \int_{0}^{x}(x-t)^{(\lceil\alpha\rceil-\alpha-1)} f_{g H}^{(\lceil\alpha\rceil)}(t) d t
$$

where $x>0$ and $\lceil\alpha\rceil=m$.

Theorem 3.2. [1] Let $f:[a, b] \rightarrow \mathbb{R}_{\mathcal{F}}$ be a fuzzy-valued function on $[a, b]$. 
(i) If $f$ is $[(i)-g H]$-differentiable at $x_{0} \in[a, b]$ then $f$ is ${ }^{C}[(i)-g H]$ - differentiable at $x_{0}$.

(ii) If $f$ is $[(i i)-g H]$-differentiable at $x_{0} \in[a, b]$ then $f$ is ${ }^{C}[(i i)-g H]$-differentiable at $x_{0}$.

Lemma 3.3. [1] The Fuzzy Fractional Initial Value Problem (FFIVP)

$$
{ }_{g H}^{C} D^{\alpha}(x(t))=f(t, x(t)), \quad x(0)=x_{0}, \quad t \in[0, a],
$$

for which $0<\alpha<1$, is equivalent to one of the following integral equations

$$
x(t)=x(0)+\frac{1}{\Gamma(\alpha)} \int_{0}^{t}(s-t)^{\alpha} f(s, x(s)) d s,
$$

or

$$
x(t)=x(0) \ominus(-1) \frac{1}{\Gamma(\alpha)} \int_{0}^{t}(s-t)^{\alpha} f(s, x(s)) d s .
$$

Here, we introduce and define a new metric on space $\mathbb{R}_{\mathcal{F}}$

$$
d_{\infty}^{\lambda}(u, v)=\max _{t \in[0, a]} \frac{d_{\infty}(u(t), v(t))}{E_{\alpha}\left(\lambda t^{\alpha}\right)}, \quad \alpha>0, \lambda>0, u, v \in \mathbb{R}_{\mathcal{F}} .
$$

Lemma 3.4. Assume $d_{\infty}(u, v)$ is Hausdorff metric on $\mathbb{R}_{\mathcal{F}}$.

(i) $d_{\infty}^{\lambda}$ is a metric on space of $C\left([0, a], \mathbb{R}_{\mathcal{F}}\right)$.

(ii) The metric $d_{\infty}^{\lambda}$ is equivalent to the metric $d_{\infty}^{0}=\max _{t \in[0, a]} d_{\infty}(x(t), y(t))$.

(iii) The metric space $\left(\left(C[0, a], \mathbb{R}_{\mathcal{F}}\right), d_{\infty}^{\lambda}\right)$ is complete.

Proof. (i) If $\lambda>0$ is constant, then we have $E_{\alpha}\left(\lambda t^{\alpha}\right)>0$ for all $t \in[0, a]$ and $E_{\alpha}$ is continuous on $[0, a]$. Therefore, three properties of a metric can be easily verified.

(ii) Since $E_{\alpha}$ is continuous and strictly increasing on $[0, a]$, we have

$$
\frac{1}{E_{\alpha}\left(\lambda a^{\alpha}\right)} \leq \frac{1}{E_{\alpha}\left(\lambda t^{\alpha}\right)} \leq 1, \quad \text { for all } \quad t \in[0, a]
$$

hence, it ensures that two metrics $d_{\infty}^{\lambda}$ and $d_{\infty}^{0}$ are equivalent.

(iii) The completeness of $\left(C[0, a], d_{\infty}^{\lambda}\right)$ follows from the completeness of $\left(C[0, a], d_{\infty}^{0}\right)$ (see $\left.[10]\right)$ and (ii). If $\left\{x_{n}\right\}$ is a Cauchy sequence in $C\left([0, a], d_{\infty}^{0}\right)$, thus (ii) follows that $\left\{x_{n}\right\}$ is a Cauchy sequence in $C\left([0, a], d_{\infty}^{\lambda}\right)$ and

$$
\lim _{n, m \rightarrow \infty} d_{\infty}^{0}\left(x_{n}, x_{m}\right)=0, \quad \text { implies } \quad \lim _{n, m \rightarrow \infty} d_{\infty}^{\lambda}\left(x_{n}, x_{m}\right)=0 .
$$

Q.E.D.

Lemma 3.5. [20]For every constant $\lambda>0$, we have

$$
\frac{1}{\Gamma(\alpha)} \int_{0}^{t}(t-s)^{\alpha-1} E_{\alpha}\left(\lambda s^{\alpha}\right) d s=\frac{E_{\alpha}\left(\lambda t^{\alpha}\right)-1}{\lambda} \quad \text { for all } t \geq 0 .
$$


Theorem 3.6. Let $R_{0}=[0, a] \times \mathbb{R}_{\mathcal{F}}$ and $f: R_{0} \rightarrow \mathbb{R}_{\mathcal{F}}$ be continuous such that the following Lipschitz condition holds: There exist a constant $L>0$ such that

$$
d_{\infty}(f(t, x), f(t, y)) \leq L d_{\infty}(x, y), \quad(t, x),(t, y) \in R_{0}
$$

Then the FFIVP

$$
{ }_{g H}^{C} D^{\alpha} x(t)=f(t, x), \quad x\left(t_{0}\right)=x_{0}
$$

has two unique solutions on $[0, a]$.

Proof. Since $f$ is a continuous function on $R_{0}$, so the integral equations (10) and (11) are well defined. Let $L>0$ be the constant defined in (15), and set $\lambda:=L \gamma$ where $\gamma>1$ is an arbitrary constant. Consider the complete metric space $\left(C[0, a], d_{\infty}^{\lambda}\right)$, and let operators $F_{1}$ and $F_{2}$ are defined by

and

$$
\left(F_{1} x\right)(t):=x(0)+\frac{1}{\Gamma(\alpha)} \int_{0}^{t}(t-s)^{\alpha-1} f(s, x(s)) d s, \quad t \in[0, a]
$$

$$
\left(F_{2} x\right)(t):=x(0) \ominus(-1) \frac{1}{\Gamma(\alpha)} \int_{0}^{t}(t-s)^{\alpha-1} f(s, x(s)) d s \quad t \in[0, a] .
$$

According to Lemma 3.3, the proof of the theorem is equivalent to show that each of two operators $F_{1}$ and $F_{2}$ have a fixed point, i.e. there exist unique points $x_{1}$ and $x_{2}$ such that $F_{1}\left(x_{1}\right)=x_{1}$ and $F_{2}\left(x_{2}\right)=x_{2}$.

So, we will prove that $F_{1}, F_{2}: C\left([0, a], \mathbb{R}_{\mathcal{F}}\right) \rightarrow C\left([0, a], \mathbb{R}_{\mathcal{F}}\right)$ are contractive maps with contraction constant $\sigma=1 / \gamma<1$, with respect to the metric $d_{\infty}^{\lambda}$.

For any $x, y \in C\left([0, a], \mathbb{R}_{\mathcal{F}}\right)$, consider

$$
\begin{aligned}
d_{\infty}^{\lambda}\left(F_{1} x, F_{1} y\right): & =\max _{t \in[0, a]} \frac{d_{\infty}\left(F_{1} x(t), F_{1} y(t)\right)}{E_{\alpha}\left(\lambda t^{\alpha}\right)} \\
& \leq \max _{t \in[0, a]}\left(\frac{1}{E_{\alpha}\left(\lambda t^{\alpha}\right)} \frac{1}{\Gamma(\alpha)} \int_{0}^{t}(t-s)^{\alpha-1} d_{\infty}(f(s, x(s)), f(s, y(s))) d s\right) \\
& \leq \max _{t \in[0, a]}\left(\frac{1}{E_{\alpha}\left(\lambda t^{\alpha}\right)} \frac{1}{\Gamma(\alpha)} \int_{0}^{t}(t-s)^{\alpha-1} L d_{\infty}(x(s), y(s)) d s\right) \\
& =L \max _{t \in[0, a]}\left(\frac{1}{E_{\alpha}\left(\lambda t^{\alpha}\right)} \frac{1}{\Gamma(\alpha)} \int_{0}^{t}(t-s)^{\alpha-1} E_{\alpha}\left(\lambda s^{\alpha}\right) \frac{d_{\infty}(x(s), y(s))}{E_{\alpha}\left(\lambda s^{\alpha}\right)} d s\right) \\
& \leq L d_{\infty}^{\lambda}(x, y) \max _{t \in[0, a]}\left(\frac{1}{E_{\alpha}\left(\lambda t^{\alpha}\right)} \frac{1}{\Gamma(\alpha)} \int_{0}^{t}(t-s)^{\alpha-1} E_{\alpha}\left(\lambda s^{\alpha}\right) d s\right) \\
& =L d_{\infty}^{\lambda}(x, y) \max _{t \in[0, a]}\left(\frac{1}{E_{\alpha}\left(\lambda t^{\alpha}\right)}\left(\frac{E_{\alpha}\left(\lambda t^{\alpha}\right)-1}{\lambda}\right)\right) \\
& =\frac{d_{\infty}^{\lambda}(x, y)}{\gamma} \max _{t \in[0, a]}\left(1-\frac{1}{E_{\alpha}\left(\lambda t^{\alpha}\right)}\right) \\
& =\frac{d_{\infty}^{\lambda}(x, y)}{\gamma}\left(1-\frac{1}{E_{\alpha}\left(\lambda a^{\alpha}\right)}\right) \\
& \leq \frac{d_{\infty}^{\lambda}(x, y)}{\gamma} .
\end{aligned}
$$


Thus, we see that the condition $\gamma>1$ ensures that $F_{1} x$ is a contractive map. Similarly we can prove that $F_{2} x$ is a contractive map, so fuzzy fractional differential equation (9) has two unique solutions.

Q.E.D.

Remark 3.7. In Theorem 3.6, we use the metric (12) that involves the Mittag-Leffler function. This particular metric is optimal in the sense that it compels the operator to be contractive on the whole of $C\left([0, a], \mathbb{R}_{\mathcal{F}}\right)$, i.e., the space of fuzzy continuous functions on $[0, a]$, rather than on a smaller set in the previous research.

For example, according to Theorem 4.3 in [1] it has been proved that a fuzzy fractional differential equation has two unique solutions on $[0, r]$, such that $0 \leq r \leq a$. In addition, the conditions of Theorem 4.3 [1] are somewhat robust whereas in this study, we only use the Lipschitz condition. In other words, the metric (12) not only help us to find two solutions on whole of $C\left([0, a], \mathbb{R}_{\mathcal{F}}\right)$ but also it allows us to have an easier proof, that is we only need to provide the condition which satisfies the well-known Banach fixed-point theorem.

Example 3.8. suppose that $f(t, y(t))=\eta y(t)$, so

$$
d_{\infty}\left(f\left(t, y_{1}(t)\right), f\left(t, y_{2}(t)\right)\right) \leq|\eta| d_{\infty}\left(y_{1}(t), y_{2}(t)\right)
$$

and $L=|\eta|$, since $\lambda:=L \gamma$ and $\gamma>1$, the fuzzy fractional differential equation ${ }_{g H} D^{\alpha} x(t)=$ $f(t, x(t)), \quad x(0)=x_{0}$, where $x_{0} \in \mathbb{R}_{\mathcal{F}}$, has two unique solutions on the space $C\left([0, a], \mathbb{R}_{\mathcal{F}}\right)$ with the metric $d_{\infty}^{\lambda}$ for all $\lambda>|\eta|$. The exact solution of the fuzzy fractional differential equation for $\eta>0$ in the case of ${ }^{C}[(i)-g H]$-differentiable is $x=x_{0} E_{\alpha}\left(\eta x^{\alpha}\right)$ and for $\eta<0$ in the case of ${ }^{C}[(i i)-g H]$-differentiable is $x=x_{0} E_{\alpha}\left(\eta x^{\alpha}\right)$.

Example 3.9. Assume that $f(t, y(t)):=\frac{1}{\sqrt{\pi}} y(t)+(0,1,2) t$, thus

$$
d_{\infty}\left(f\left(t, y_{1}(t)\right), f\left(t, y_{2}(t)\right)\right) \leq \frac{1}{\sqrt{\pi}} d_{\infty}\left(y_{1}(t), y_{2}(t)\right)
$$

and $L=\frac{1}{\sqrt{\pi}}$, since $\lambda:=L \gamma$ and $\gamma>1$, the fuzzy fractional differential equation ${ }_{g H}{ }^{C} D^{\alpha} x(t)=$ $f(t, x(t)), \quad x(0)=[-0.1262 r,-0.2523+0.1262 r], t \in\left[0, \frac{\pi}{2}\right]$, has two unique solutions on the space $C\left(\left[0, \frac{\pi}{2}\right], \mathbb{R}_{\mathcal{F}}\right)$ with the metric $d_{\infty}^{\lambda}$ for all $\lambda>\frac{1}{\sqrt{\pi}}$. For example, when $\alpha=1$, the exact solution in the case of ${ }^{C}[(i)-g H]$-differentiable is,

$$
\begin{gathered}
\underline{x}=-\pi^{2} r-e^{\frac{t}{\pi}}\left(\frac{631 r}{5000}-\pi^{2} r\right)-\pi r t, \\
\bar{x}=e^{\frac{t}{\pi}\left(\frac{631 r}{5000}-\pi^{2} r+2 \pi^{2}-\frac{2523}{10000}\right)-2 \pi t+\pi^{2} r-2 \pi^{2}+\pi r t}
\end{gathered}
$$

and in the case of ${ }^{C}[(i i)-g H]$-differentiable is

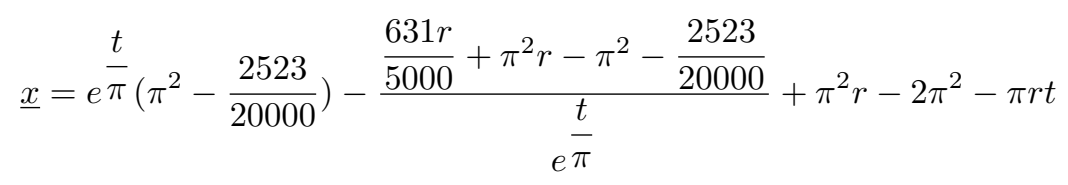




$$
\bar{x}=\frac{\left(\frac{631 r}{5000}+\pi^{2} r-\pi^{2}-\frac{2523}{20000}\right)}{e^{\frac{t}{\pi}}}-2 \pi t+e^{\frac{t}{\pi}}\left(\pi^{2}-\frac{2523}{20000}\right)-\pi^{2} r+\pi r t .
$$

\section{References}

[1] T. Allahviranloo, A. Armand and Z. Gouyandeh, Fuzzy fractional differential equations under generalized fuzzy Caputo derivative. Journal of Intelligent \& Fuzzy Systems. 26 (2014), 14811490.

[2] S. Arshad, on existence and uniqueness of solution of fuzzyfractional differential equations. Iranian Journal of Fuzzy Systems. Vol. 10. No. 6 (2013), 137-151.

[3] D. Baleanu, K. Diethelm, E. Scalas and J. J. Trujillo, Fractional Calculus Models and Numerical Methods, in: Series on Complexity, Nonlinearity and Chaos. World Scientific, Singapore, (2012).

[4] B. Bede, Mathematics of fuzzy sets and fuzzy logic. Springer-Verlag Berlin Heidelberg (2013).

[5] B. Bede and S. G. Gal, Generalizations of the differentiability of fuzzy-number-valued functions with applications to fuzzy differential equations. Fuzzy Sets and Systems, 151 No. 3 (2005), 581-599.

[6] B. Bede, I. J. Rudas and A. L. Bencsik, First order linear fuzzy differential equations under generalized differentiability. Information Sciences. 177 (2007), 1648-1662.

[7] B. Bede and L. Stefanini, Generalized differentiability of fuzzy-valued functions. Fuzzy Sets and Systems. 230(1) (2013), 119-114, doi: 10.1016/j.fss.2012.10.003.

[8] K. Diethelm: The Analysis of Fractional Differential Equations. Springer, Heidelberg (2010).

[9] R. Hilfer, Applications of Fractional Calculus in Physics. Academic Press, Orlando, (1999).

[10] , O. Kaleva Fuzzy differential equations. Fuzzy Sets and Systems. 24 (1987), 301-317.

[11] A. A. Kilbas, H. M. Srivastava and J. J. Trujillo, Theory and applications of fractional differential equations. North-Holland Mathematics Studies, 204 (2006), Elsevier, Amsterdam.

[12] F. C. Meral, T. J. Royston and R. Magi, Fractional calculus in viscoelasticity: an experimental study. Commun Nonlinear Sci Numer Simulat. 15(4) (2010), 939-45.

[13] K. B. Oldham, Fractional differential equations in electrochemistry. Advances in Engineering Software. 41 (2010), 9-12.

[14] I. Podlubny, Fractional Differential Equations. Academic Press, San Diego, (1999).

[15] M. L. Puri and D. A. Ralescu, Differentials of fuzzy functions. J. Math. Anal. Appl. 91(2) (1983), 552-558. 
[16] S. Salahshour, T. Allahviranloo, S. Abbasbandy and D. Baleanu, Existence and uniqueness results for fractional differential equations with uncertainty. Advances in Difference Equations 2012, 112 (2012), doi:10.1186/1687-1847-2012-112.

[17] S. Song and C. Wu, Existence and uniqueness of solutions to Cauchy problem of fuzzy differential equations. Fuzzy Sets and Systems. 110 (2000), 55-67.

[18] A. M. Spasic and M. P. Lazarevic, Electroviscoelasticity of liquid/liquid interfaces: fractionalordermodel, J. Colloid Interface Sci. 282 (2005), 223-230.

[19] L. Stefanini, A generalization of Hukuhara difference for interval and fuzzy arithmetic. Fuzzy Sets and Systems. 161 (2010), 1564-1584.

[20] C. C. Tisdell, When do fractional differential equations have solutions that are bounded by the Mittag-Leffler function?. Fractional Calculus \& Applied Analysis. 18(3) (2015), 642-650, doi: 10.1515/fca-2015-0039. 